\title{
Eleven synchronous/ metachronous primary carcinomas of the upper gastrointestinal tract: a case report
}

\author{
Shogo Shinohara ${ }^{1}$, Masahiro Kikuchi ${ }^{1}$, Risa Tona ${ }^{2}$, I ppei Kishimoto ${ }^{1}$, Hiroyuki Harada ${ }^{1}$, Naoto \\ Shimeno $^{3}$
}

1. Department of Otolaryngology-Head and Neck Surgery, Kobe City Medical Center, Minatojima-minamimachi, Chuoku, Kobe, Japan. 2. Department of Otolaryngology, Institute of Biomedical Research and Innovation, Minatojimaminamimachi, Chuo-ku, Kobe, Japan. 3. Department of Gastrointestinal Medicine, Kobe City Medical Center General Hospital, Minatojima-minamimachi, Chuo-ku, Kobe, Japan.

Correspondence: Shogo Shinohara. Address: Department of Otolaryngology-Head and Neck Surgery, Kobe City Medical Center General Hospital, Minatojima-minamimachi 2-1-1, Chuo-ku, Kobe, 650-0047, Japan. E-mail: sinosino@kcho.jp

Received: May 30, 2014

Accepted: July 14, 2014

Online Published: July 23, 2014

DOI : $10.5430 /$ crcp.v1n2p159

URL: http://dx.doi.org/10.5430/crcp.v1n2p159

\begin{abstract}
In this paper, we report a patient with eleven synchronous/metachronous carcinomas in the upper gastrointestinal (UGI) tract treated successfully for five years. A 65-year-old male with a history of heavy smoking first visited our hospital with abnormal stomach findings by UGI endoscopy. The patient was a heavy drinker and often showed facial flushing in his younger years after drinking a glass of beer; thus, it was hypothesized that he had inactive heterogeneous aldehyde dehydrogenase 2 alleles. Biopsy revealed the existence of adenocarcinoma of the stomach and endoscopic submucosal dissection (ESD) was subsequently performed. During the operation, three more cancers were found: two in the esophagus and one in the left pyriform sinus. Esophageal lesions were removed simultaneously by ESD. Endoscopic mucosal resection of a hypopharyngeal carcinoma using a curved rigid esophagoscope was performed one month following diagnosis. At the subsequent follow-up, metachronous squamous cell carcinomas in the right pyriform sinus (fifth), posterior wall of oropharynx (sixth), oral floor (seventh), esophagus (eighth and ninth), posterior wall of hypopharynx (tenth), and soft palate (eleventh) were detected. All lesions were pathologically and endoscopically independent and successfully removed using an endoscopic or transoral technique. The patient is currently alive with no evidence of disease.
\end{abstract}

\section{Key words}

Multiple primary malignancy, Upper gastrointestinal tract, Flusher, ALDH2

\section{I ntroduction}

Routine follow-up using an upper gastrointestinal (UGI) endoscope is recommended for patients who have had a previously treated UGI cancer based on the "field cancerization" theory ${ }^{[1]}$. Approximately $40 \%$ of Japanese individuals who show past or current facial flushing after consuming small doses of alcohol (flusher) are likely to have inactive heterozygous aldehyde dehydrogenase-2 (ALDH2) and a subsequent higher relative risk of developing alcohol-related UGI tract cancers compared with individuals with wild-type ALDH2 alleles ${ }^{[2,3]}$. Recently, endoscopic technology innovations have permitted the identification of very small, superficial carcinomas in the UGI tract using magnifying 
endoscopy or endoscopy with narrow band imaging (NBI) ${ }^{[4,5]}$. In this case report, we present a case of a former flusher with eleven independent synchronous/metachronous carcinomas in the UGI that were found on routine follow-up and successfully removed before clinical manifestation.

\section{Case report}

A 65-year-old male first visited our hospital with abnormal findings in the stomach after routine UGI endoscope check-up. He was an ex-smoker who used to smoke one pack per day for 45 years. The patient also used to experience facial flushing when drinking when he was younger but has since become a heavy drinker with a total daily alcohol consumption of over $80 \mathrm{~g}$. After making the pathological diagnosis of adenocarcinoma of the stomach (first carcinoma), the tumor was removed by endoscopic submucosal dissection (ESD). During the operation, multiple Lugol-voided lesions (LVLs) were visualized by Lugol chromoendoscopy and three more superficial cancers were found: two in the esophagus (second and third carcinomas) and one in the left pyriform sinus (fourth carcinoma). Esophageal lesions were simultaneously removed by ESD (both were pathologically proven to be squamous cell carcinomas) and the hypopharyngeal lesion was biopsied for pathological diagnosis. Transoral surgery for removing the superficial squamous cell carcinoma in left pyriform sinus was performed 1 month after the ESD of the esophageal lesions. Briefly, the larynx was elevated to extend and visualize the pyriform sinus using a rigid curved esophagoscope while the patient was under general anesthesia. The region containing the carcinoma was identified by UGI scope with NBI and confirmed by applying Lugol dye solution. The carcinoma was successfully removed in six pieces by endoscopic mucosal resection using a transparent cap.

Intimate follow-up using magnifying endoscopy or endoscopy with NBI detected metachronous squamous cell carcinomas in the right pyriform sinus (fifth), posterior wall of the oropharynx (sixth), oral floor (seventh), and esophagus (eighth) within one year of the resection of the first carcinoma. At this time, we performed bilateral neck dissections for metastasized squamous cell carcinoma in the right level three lymph node, the primary lesion of which was unknown. After the neck dissection, we administered oral fluorouracil (S-1: $80 \mathrm{mg} / \mathrm{m}^{2} /$ day on days 1-14) every four weeks for one year. While undergoing chemotherapy treatment, no new lesions were identified during any of the monthly surveys. Fourteen months after completion of the adjuvant chemotherapy, UGI endoscopy revealed a new superficial carcinoma in the esophagus (ninth). Two more carcinomas were identified within 1 year: posterior wall of the hypopharynx (tenth) and the soft palate (eleventh). All new lesions were successfully removed by endoscopic or transoral techniques. The table shows the organs, lesions, and time after resection of the first carcinoma, pathological diagnoses, and pT stage (UICC 7th) of all carcinomas. The figure illustrated the ordinary or lugol-endoscopic findings, NBI endoscopic findings and pathological findings of all eleven carcinomas and metastatic lymphnnode. The patient is currently alive with no evidence of disease.

Figure. Endoscopic and pathological findings of all eleven primary carcinomas and metastatic lymph node: (upper line; ordinary endoscopic or lugol-chromoendoscopic findings, middle line; NBI endoscopic findings, lower line; pathological findings)

Note. N/A: not available
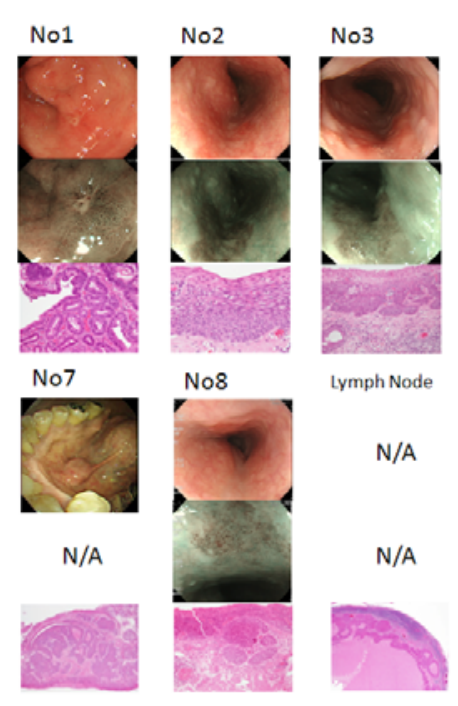

Lymph Node

N/A

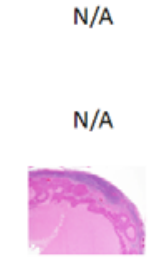

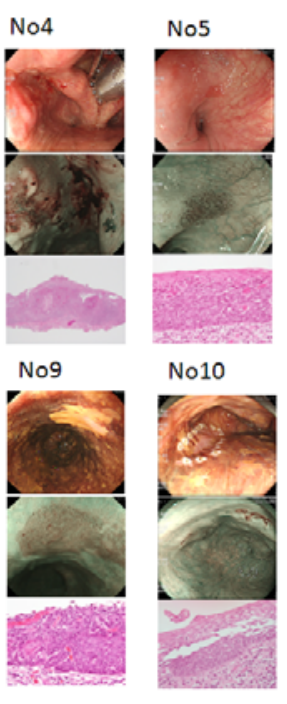

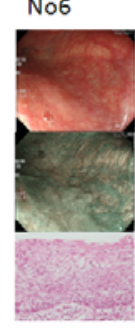

No11

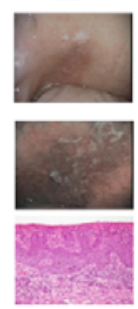


Table. Descriptions of the patient's synchronous/metachronous primary carcinomas

\begin{tabular}{lllll}
\hline Number & Organ & Lesion & Months & Pathology, pT stage \\
\hline 1 & stomach & angular incisure & 0 & Adenocarcinoma T1 \\
2 & esophagus & DL29cm, posterior & 1 & SCC T1a \\
3 & esophagus & DL27cm, posterior & 1 & SCC T1a \\
4 & hypopharynx & right pyriform sinus & 2 & SCC T1 \\
5 & hypopharynx & left pyriform sinus & 6 & CIS \\
6 & posterior wall & 6 & CIS \\
7 & oropharynx & anterior oral floor & 7 & SCC T1 \\
8 & oral cavity & DL27cm, anterior & 8 & SCC T1a \\
- & esophagus & right neck level 3 & 12 & metastatic SCC \\
9 & lymphnode & DL38cm, anterior & 39 & SCC T1a \\
10 & esophagus & left pyriform sinus & 41 & CIS \\
11 & hypopharynx & right side of soft palate & 50 & SCC T1 \\
\hline
\end{tabular}

Note. Months: Months after the resection of the first carcinoma; DL: Dental line; SCC: Squamous cell carcinoma; CIS: Carcinoma in situ

\section{Discussion}

Patients with head and neck squamous cell carcinoma (HNSCC) are known to often have or subsequently develop second primary esophageal cancers. Multiple occurrences of neoplastic changes in the UGI tract have been explained by the "field cancerization" concept ${ }^{[1]}$. In a survey of our institute from 2004 to 2010, 83 synchronous/metachronous primary carcinomas from 286 patients with newly diagnosed HNSCC (29\%) were identified; of these, synchronous primary carcinomas were most frequently found in the esophagus (46\%) ${ }^{[6]}$. Multiple occurrences of head and neck and UGI tract malignancies are usually associated with repeated exposure to carcinogens, such as alcohol and tobacco. In the present case, the patient previously smoked one pack of cigarettes per day for 45 years, which likely had a carcinogenic influence on UGI mucosal epithelium. Moreover, he was a habitual drinker ingesting over $80 \mathrm{~g}$ of alcohol daily, and we hypothesize that he had inactive heterogeneous ALDH2 alleles because he had a previous tendency to develop facial flushing immediately after drinking a glass of beer ${ }^{[2]}$. The simple questionnaire asking past or current facial flushing after small dose of alcohol was reported to have an $89 \%$ sensitivity and $90 \%$ specificity for screening inactive heterogeneous ALDH2 ${ }^{[2]}$. Mutant alleles encoding an inactive subunit of ALDH2 is almost exclusively carried by East Asian people and about $40 \%$ of Japanese people have inactive ALDH2 alleles, $7 \%$ of which are homozygous and 35\% heterozygous ${ }^{[3,7,8]}$. After drinking $0.1 \mathrm{~g}$ ethanol $/ \mathrm{kg}$ bodyweight, individuals with homozygous inactive ALDH2 and heterozygous inactive ALDH2 have 18 and five times higher peak blood acetaldehyde concentrations, respectively, than individuals with homozygous active ALDH2 ${ }^{[9]}$. Heavy drinkers with either homozygous or heterozygous inactive alleles are reported to be highly susceptible to esophageal cancers and head and neck cancers with odds ratios of 10.4 and 3.57, respectively, compared with drinkers with homozygous active ALDH2 ${ }^{[10]}$. Multiple esophageal LVLs identified using Lugol chromoendoscopy were observed in the present case, which are reported to be found in $80 \%$ of drinkers with inactive ALDH2 ${ }^{[11,12]}$. The identification of these lesions prompted a routine survey using a rhino-laryngo fiberscope and a UGI endoscope. A cervical lymph node metastasis appeared one year after the resection of the first carcinoma, but it was not possible to determine which of the primary carcinomas was responsible for this. After resection of the lymph node metastasis, we started adjuvant chemotherapy using oral fluorouracil and continued treatment for one year, the course of which was uneventful. Radiation therapy was not an option not only because all lesions were well controlled using a surgical approach, but also because radiation would make endoscopic observations using NBI difficult. We also intended to reserve radiation for future use in case new UGI tract carcinomas arose that were unresectable by endoscopy and yielded clinical symptoms. 


\section{Conflict of interests}

The authors report no conflict of interests in this work.

\section{Acknowledgments}

This study was financially supported by the Kasahara Fund for the Promotion of Cancer Research.

\section{References}

[1] Slaughter DP, Southwick HW, Smejkal W. Field cancerization in oral stratified squamous epithelium; clinical implications of multicentric origin. Cancer. 1953; 6: 963-968.

http://dx.doi.org/10.1002/1097-0142(195309)6:5<963::AID-CNCR2820060515>3.0.CO;2-Q

[2] Yokoyama T, Yokoyama A, Kato H, Tsujinaka T, Muto M, Omori T, et al. Alcohol flushing, alcohol and aldehyde dehydrogenase genotypes, and risk for esophageal squamous cell carcinoma in Japanese men. Cancer Epidemiol Biomarkers Prev. 2003; 12: 1227-1233. PMid: 14652286.

[3] Higuchi S, Matsushita S, Murayama M, Takagi S, Hayashida M. Alcohol and aldehyde dehydrogenase polymorphisms and the risk for alcoholism. Am J Psychiatry. 1995; 152: 1219-1221. PMid: 7625477.

[4] Yoshida T, Inoue H, Usui S, Satodate H, Fukami N, Kudo SE. Narrow-band imaging system with magnifying endoscopy for superficial esophageal lesions. Gastrointest Endosc. 2004; 59: 288-295. http://dx.doi.org/10.1016/S0016-5107(03)02532-X

[5] Nakayoshi T, Tajiri H, Matsuda K, Kaise M, Ikegami M, Sasaki H. Magnifying endoscopy combined with narrow band imaging system for early gastric cancer: correlation of vascular pattern with histopathology (including video). Endoscopy. 2004; 36: 1080-1084. PMid: 15578298. http://dx.doi.org/10.1055/s-2004-825961

[6] Tona R, Shinohara S, Kikuchi M, Fujiwara K, Yamazaki H, Kishimoto I, et al. Synchronous multiple malignancies in patients with head and neck squamous cell carcinoma. Practica Oto-Rhino-Laryngologica. 2013; 106: 155-160. http://dx.doi.org/10.5631/jibirin.106.155

[7] Harada S, Agarwal DP, Goedde HW, Tagaki S, Ishikawa B. Possible protective role against alcoholism for aldehyde dehydrogenase isozyme deficiency in Japan. Lancet. 1982; 2: 827. http://dx.doi.org/10.1016/S0140-6736(82)92722-2

[8] Harada S, Agarwal DP, Goedde HW, Ishikawa B. Aldehyde dehydrogenase isozyme variation and alcoholism in Japan. Pharmacol Biochem Behav. 1983; 18 Suppl 1: 151-153. http://dx.doi.org/10.1016/0091-3057(83)90163-6

[9] Enomoto N, Takada A, Date T. Genotyping of the aldehyde dehydrogenase 2 (ALDH2) gene using the polymerase chain reaction: evidence for single point mutation in the ALDH2 gene of ALDH2-deficiency. Gastroenterol Jpn. 1991; 26: 440-447. PMid: 1916152.

[10] Yokoyama A, Omori T, Yokoyama T. Alcohol and aldehyde dehydrogenase polymorphisms and a new strategy for prevention and screening for cancer in the upper aerodigestive tract in east asians. Keio J Med. 2010; 59: 115-130. PMid: 21187698. http://dx.doi.org/10.2302/kjm.59.115

[11] Muto M, Hitomi Y, Ohtsu A, Ebihara S, Yoshida S, Esumi H. Association of aldehyde dehydrogenase 2 gene polymorphism with multiple oesophageal dysplasia in head and neck cancer patients. Gut. 2000; 47: 256-261. PMid: 10896918. http://dx.doi.org/10.1136/gut.47.2.256

[12] Muto M, Nakane M, Hitomi Y, Yoshida S, Sasaki S, Ohtsu A, et al. Association between aldehyde dehydrogenase gene polymorphisms and the phenomenon of field cancerization in patients with head and neck cancer. Carcinogenesis. 2002; 23: 1759-1765. PMid: 12376487. http://dx.doi.org/10.1093/carcin/23.10.1759 